\title{
Autorinnen, Autoren, Herausgeberin und Herausgeber
}

Florian Aschinger, Diplom-Soziologe, studierte Soziologie mit dem Schwerpunkt empirische Sozialforschung in Bamberg und Bilbao. Er arbeitete als Bildungsforscher im Projekt „Nationales Bildungspanel“ (NEPS) am Leibniz-Institut für Bildungsverläufe (LIfBi e.V.) in Bamberg, vorwiegend im Bereich der Hochschulforschung, und unterrichtete qualitativ orientierte Methoden der empirischen Sozialforschung sowie Mixed Methods-Ansätze an den Universitäten Bamberg und Kassel. Im Projekt Lernwelt Hochschule war er für die Konzeption, Durchführung und Auswertung der Leitfadeninterviews mit Hochschulakteurinnen und -akteuren verantwortlich. Kontakt: florian@aschinger.net.

Alexandra Becker (M. A.) ist an der Hochschule der Medien Stuttgart (HdM) im Learning-Research Center tätig. Seit 2012 befasst sie sich mit den Themen physische Lernräume, Selbstlernzentren und Hochschulorganisation. So leitet sie die Forschungen zum Selbstlernzentrum Lernwelt der Hochschule der Medien. Ein weiterer Schwerpunkt liegt bei ihr im Bereich der Hochschulforschung, vor allem im Hinblick auf die Entwicklungen zur studierendenorientierten Hochschule. Sie leitete das Projekt Lernwelt Hochschule. Kontakt: beckera@hdm-stuttgart. de.

Fabian Franke, Dr., war nach dem Studium der Physik und dem Bibliotheksreferendariat von 1998 bis 2006 als Fachreferent und Leiter des Informationszentrums an der Universitätsbibliothek Würzburg tätig. Seit 2006 leitet er die Universitätsbibliothek Bamberg. Er ist Mitglied des Standing Committee Information Literacy des internationalen Bibliotheksverbands IFLA und der Arbeitsgruppe Informationskompetenz des Bibliotheksverbunds Bayern. Von 2012 bis 2018 war er Vorsitzender der Gemeinsamen Kommission Informationskompetenz des Deutschen Bibliotheksverbands und des Vereins Deutscher Bibliothekarinnen und Bibliothekare. Er koordinierte das Forschungsprojekt Lernwelt Hochschule an der Otto-Friedrich-Universität Bamberg. Kontakt: fabian.franke@uni-bamberg.de.

Nicole Gageur schloss ihr Philosophie- und Germanistikstudium mit dem Magister ab. Während ihres Studiums und danach arbeitete sie in einer Unternehmensberatung und als freie Journalistin. Sie absolvierte an der Hochschule für Angewandte Wissenschaften in Hamburg ihr Zweitstudium „Bibliotheks- und Informationsmanagement“ und arbeitete dort anschließend als wissenschaftli-

๖ Open Access. () 2020 Richard Stang, published by De Gruyter. (ब्C) Br-Nc-ND This work is licensed under the Creative Commons Attribution-NonCommercial-NoDerivatives 4.0 License. 
che Mitarbeiterin in verschiedenen Projekten. Im Forschungsprojekt Lernwelt Hochschule bearbeitete sie unter anderem die Fallstudien sowie den LeHo-Blog. Kontakt: nicole.gageur@uni-hamburg.de.

Christine Gläser ist seit 2008 Professorin für Informationsdienstleistungen, elektronisches Publizieren, Metadaten und Datenstrukturierung an der Hochschule für Angewandte Wissenschaften (HAW) Hamburg. Aktuelle Lehr- und Forschungsschwerpunkte: Lernraum Hochschule, Teaching/Learning Library, Bibliotheksethnografie, Forschungsdatenmanagement, Data Literacy. Lernraumentwicklungen beschäftigen sie seit Anfang der 2000er Jahre. Sie bearbeitet die Thematik im Rahmen ihrer Professur in Forschung und Lehre der HAW. In der Arbeitsgruppe Lernräume der Deutschen Initiative für Netzwerkinformation e. V. (DINI) ist sie seit 2009 aktiv. Für das Projekt Lernwelt Hochschule hatte sie an der Hochschule für Angewandte Wissenschaften (HAW) Hamburg die koordinierende und konzeptionelle Verantwortung. Kontakt: Christine.Glaeser@haw-hamburg.de.

Laura Kobsch schloss das Bachelorstudium „Bibliotheks- und Informationsmanagement“ und das Masterstudium „Information, Medien, Bibliothek“ an der Hochschule für Angewandte Wissenschaften (HAW) Hamburg ab. Von Januar bis September 2019 war sie als wissenschaftliche Mitarbeiterin im Forschungsprojekt Lernwelt Hochschule tätig und bearbeitete unter anderem die Fallstudien sowie den LeHo-Blog. Seit Oktober 2019 ist sie wissenschaftliche Mitarbeiterin an der Hochschule Fresenius.

Anke Petschenka, Dr., ist seit 2006 wissenschaftliche Mitarbeiterin in der Universitätsbibliothek Duisburg-Essen. Sie ist bibliotheksfachliche Ansprechpartnerin für diverse Fachdisziplinen und für den Bereich E-Learning in der UB zuständig. Sie ist zudem Mitglied in der E-Learning-Allianz der Universität Duisburg-Essen sowie im Moodle-Kompetenzzentrum. Seit März 2018 ist sie Sprecherin der Arbeitsgruppe Lernräume der Deutschen Initiative für Netzwerkinformation e. V. (DINI). In ihrer Masterarbeit des berufsbegleitenden MALIS-Studiengangs an der Technischen Hochschule Köln hat sie sich 2013 mit der Gestaltung virtueller Lernräume beschäftigt. Ihre Promotion hat sie 2005 zum Thema Kommunikationsprozesse in netzbasierten Lernszenarien an der Universität Duisburg-Essen eingereicht. Sie koordinierte seit 2018 das Forschungsprojekt Lernwelt Hochschule auf Seiten der Deutschen Initiative für Netzwerkinformation e. V. (DINI). Kontakt: anke.petschenka@uni-due.de. 
Richard Stang, Dr. phil., Diplom-Pädagoge und Diplom-Soziologe, ist Professor für Medienwissenschaft im Studiengang „Informationswissenschaften“ in der Fakultät „Information und Kommunikation“ der Hochschule der Medien Stuttgart (HdM). Er leitet das Learning Research Center der Hochschule der Medien Stuttgart gemeinsam mit Prof. Dr. Frank Thissen (www.learning-research.center). Arbeitsschwerpunkte sind unter anderem Lernwelten, Bildungsforschung, Medienentwicklung und Innovationsforschung. Er berät Kommunen und Einrichtungen (Hochschulen, Bibliotheken usw.) bei der Gestaltung von Lernwelten. Im Forschungsprojekt Lernwelt Hochschule hatte er die wissenschaftliche Gesamtleitung inne. Kontakt: stang@hdm-stuttgart.de.

Hans-Dieter Weckmann studierte Mathematik und Informatik in Bonn. Seit 1977 arbeitete er als wissenschaftlicher Mitarbeiter im Rechenzentrum der Universität Duisburg, das er seit 1992 leitete. Nach der Fusion mit der Universität Essen war er seit 2006 im Zentrum für Informations- und Mediendienste verantwortlich für den Geschäftsbereich IT-Infrastruktur. Seit 2011 leitete er das Zentrum für Informations- und Medientechnologie der Heinrich-Heine-Universität Düsseldorf. In EUNIS (European University Information Systems Organisation) arbeitete er von 2006 bis 2015 im Board of Directors. Im ZKI (Zentren für Kommunikationsverarbeitung in Forschung und Lehre) war er von 2005 bis 2017 Sprecher des Arbeitskreises Universitätsrechenzentren. In der Deutschen Initiative für Netzwerkinformation e. V. (DINI) vertrat er bis 2018 die Arbeitsgruppe Lernräume und war von 2017 bis 2018 Mitglied des Vorstands. Seit 2018 ist er im Ruhestand. Er koordinierte bis 2018 das Forschungsprojekt Lernwelt Hochschule auf Seiten der Deutschen Initiative für Netzwerkinformation e. V. (DINI). Kontakt: hd.weckmann@hhu.de.

Hannes Weichert studierte Bibliotheks- und Informationsmanagement an der Hochschule der Medien Stuttgart (HdM) und durchläuft derzeit das Forschungsmasterprogramm „Master of Media Research“. Seit Juni 2018 ist er als wissenschaftlicher Mitarbeiter im Forschungsprojekt Lernwelt Hochschule tätig. Innerhalb des Forschungsprojekts beschäftigte er sich insbesondere mit der Analyse strategischer Hochschul-Dokumente und den seitens der Träger gesetzten Rahmenbedingungen. Kontakt: weichert@hdm-stuttgart.de.

Bert Zulauf studierte Elektrotechnik mit dem Schwerpunkt Prozessinformatik an der Bergischen Universität Wuppertal und Management (MBA) an der Düsseldorf Business School der Heinrich-Heine-Universität Düsseldorf. Ab 2005 verantwortete es als Abteilungsleiter des Zentrums für Informations- und Medienverarbeitung die Einrichtung des damals neuen Bereichs E-Learning an der Ber- 
gischen Universität Wuppertal. Seit Februar 2015 leitet er die Abteilung „Multimedia und Anwendungssysteme“ im Zentrum für Informations- und Medientechnologie der Heinrich-Heine-Universität Düsseldorf zu der auch der Bereich E-Learning und das Multimediazentrum der Universität gehört. Er leitete aktuell das BMBF Projekt „Forschungsdatenmanagement in Kooperation“ (FoDaKo) der Universitäten Düsseldorf, Siegen und Wuppertal, mit einem Schwerpunkt im Bereich Schulungen in Düsseldorf. Den studentischen DINI-Wettbewerb „Lehren und Lernen mitgestalten - Studieren im digitalen Zeitalter“ begleitete er als Gutachter und ist Sprecher der Arbeitsgruppe E-Learning der Deutschen Initiative für Netzwerkinformation e. V. (DINI). Er koordinierte das Forschungsprojekt Lernwelt Hochschule an der Heinrich-Heine-Universität Düsseldorf. Kontakt: zulauf@hhu.de. 